\title{
1 Identification of Langerhans-like cells in the immunocompetent tissues of channel catfish,
}

2 Ictalurus punctatus

3

4

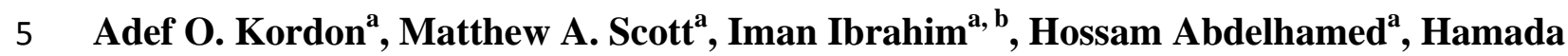

6 Ahmed $^{\mathrm{a}, \mathrm{c}}$, Wes Baumgartner ${ }^{\mathrm{d}}$, Attila Karsi ${ }^{\mathrm{a}}$, and Lesya M. Pinchuk ${ }^{\mathrm{a}, *}$

7

8

$9 \quad{ }^{a}$ Department of Basic Sciences, College of Veterinary Medicine, Mississippi State University,

10 Mississippi State, MS 39762, United States of America

$11{ }^{\mathrm{b}}$ Department of Pathology, Faculty of Veterinary Medicine, Mansoura University, Mansoura,

12 Egypt

$13{ }^{\mathrm{c}}$ Department of Nutrition and Veterinary Clinical Nutrition, Faculty of Veterinary Medicine,

14 Damanhour University, Egypt

$15{ }^{\mathrm{d}}$ Department of Pathobiology and Population Medicine, Collage of Veterinary Medicine,

16 Mississippi State University, Mississippi State, MS 39762, United States of America

17

18

19

20

21

22

23

$24 *$ Corresponding author. Department of Basic Sciences, College of Veterinary Medicine,

25 Mississippi State University, 240 Wise Center Drive, Mississippi State, MS 39762-6100, United

26 States of America.

27 E-mail addresses: pinchuk@cvm.msstate.edu (L. M. Pinchuk).

28 
Dendritic cells (DCs) are the most powerful antigen presenting cells (APCs) that have a critical role in bridging innate and adaptive immune responses in vertebrates. Dendritic cells have been

32 characterized morphologically and functionally in the teleost fish models such as rainbow trout, salmonids, medaka, and zebrafish. The presence of DCs with remarkable similarities to human Langerhans cells (LCs) has been described in the spleen and anterior kidney of salmonids and rainbow trout. However, there is no evidence of the presence of DCs and their role in channel catfish immunity. In this study, we assessed DC-like cells in the immunocompetent tissues of channel catfish by immunohistochemistry (IHC), flow cytometry and transmission electron microscopy (TEM). We identified Langerin/CD207 ${ }^{+}\left(\mathrm{L} / \mathrm{CD} 207^{+}\right)$cells in the channel catfish anterior kidney, spleen and gill by IHC. Moreover, we described the cells that resembled mammal LC DCs containing Birbeck-like (BL) granules in channel catfish spleen, anterior and posterior kidneys and gill by TEM. Our data suggest that cells with DC-like morphology in the immune related organs of catfish may share morphological and functional properties with previously reported DCs in teleost fish and mammals. More detailed knowledge of the phenotype and the function of catfish DCs will not only help gain insight into the evolution of the vertebrate adaptive immune system but will also provide valuable information for development and optimization of immunotherapies and vaccination protocols for aquaculture use.

47 Key words: Channel catfish, dendritic cells, Langerhans cells, Birbeck granules

\section{Introduction}

Dendritic cells (DCs) are the most powerful professional antigen presenting cells (APCs) that bridge, initiate, regulate, and control all innate and adaptive immune responses in vertebrates

51 [1]. Although numerous studies are still mainly focused on mammalian DCs, reports on the 
52 morphological and functional characterization of DCs in teleost fish, the earliest vertebrates to

53 develop functional adaptive immune responses, are scarce. Recently, DC-like cells, with T cell

54 stimulatory capacities that revealed the properties of mature mammalian DCs have been

55 identified in zebrafish (Danio rerio) [2, 3]. Furthermore, functional cells with dendritic

56 morphology, motility, phagocytic ability and strong $\mathrm{T}$ cell stimulatory properties have been

57 identified in several other teleost fish species such as rainbow trout (Oncorhynchus mykiss) and medaka (Oryzias latipes) $[4,5]$. Co-stimulation is an important function of professional APCs to

59 prime naïve T cells. The major co-stimulatory molecules such as CD80/CD86 and CD83 have

60 been reported in zebrafish [6]. Shao et al. reported the functional conservation of surface

61 phenotypic molecules on DCs in teleost fish as in mammals by demonstrating functional abilities

62 of $\mathrm{CD} 80 / 86 / \mathrm{CD} 83 / \mathrm{CD} 209^{+}$cells in teleost fish to promote $\mathrm{CD} 4^{+}$naïve $\mathrm{T}$ cell activation [7]. Langerhans cells (LCs) are a distinct population of immature DCs in the epidermis of

64 mammals, and this unique location at a barrier surface provides them with early access to skin 65 pathogens, commensal organisms, foreign chemicals and epidermal self-antigens [8]. Langerhans 66 cells play a sentinel role through their specialized function in antigen uptake and capture, their 67 capacity to migrate to the secondary lymphoid organs and present antigens to specific T cells thus initiating acquired immune responses [9]. Langerhans cells are uniquely characterized by

69 Birbeck granules (BGs), the organelles consisting of superimposed and zippered membranes. It was previously shown that Langerin is constitutively associated with BGs and is a potent inducer

71 of membrane superimposition [10]. Induction of BGs is a consequence of the antigen capture

72 function of Langerin, allowing routing into these organelles and providing access to a non-

73 classical antigen processing pathway [11]. But how BGs influence the processing and

74 presentation of antigens by MHC class I and class II is still not fully understood. A recent report 
75 showed that LCs mediated and enhanced cross-presentation when antigen was delivered through 76 Langerin [12].

77 Several morphological studies identified the cell type strikingly resembling mammalian LCs 78 within inflammatory gill lesions and the spleen of healthy fish based on the presence of BGs by 79 using a commercial polyoclonal antibodies (pAbs) developed against human Langerin [13-15]. 80 In particular, Langerin $/ \mathrm{CD} 207^{+}\left(\mathrm{L} / \mathrm{CD} 207^{+}\right)$cells were found in the cytoplasm of the spleen and 81 anterior kidney of Atlantic salmon (Salmo salar) and rainbow trout [14, 15]. Interestingly, BG-

82 like granules were observed in the thymus and anterior kidney followed by the appearance within 83 cells of the newly developed spleens in rainbow trout and Atlantic salmon [15]. Langerin-

84 positive cells in healthy rainbow trout were seen predominantly in the spleen however, during 85 microsporidial gill disease, the number of $\mathrm{L} / 207^{+}$cells has been significantly increased in the 86 spleen and anterior kidney [14].

87 Channel catfish (Ictalurus punctatus) is the most important aquaculture species in the United 88 States, accounting for $\$ 352$ million in 2014 [16]. Vaccination against catfish pathogens requires 89 generation of $\mathrm{T}$ and $\mathrm{B}$ cell-mediated immunity controlled by professional APCs to eliminate 90 infection and induce protective memory immune responses. Comprehensive understanding and 91 knowledge of putative DC populations in catfish is not only important for health assessment and 92 vaccine development, but also to study the evolution of the immune system. However, there is no 93 evidence of the existence of DCs and their role in orchestrating innate and adaptive immunity in 94 channel catfish. Therefore, the purpose of our research was to conduct a morphological 95 assessment of DCs in immunocompetent organs of channel catfish. Here, for the first time, we 96 report the presence of DC-like cells with the striking similarities to human LCs in the immune 97 related organs of catfish. The identification of DC-like cells in catfish suggests that specialized 
98 APCs might share properties of the mammalian DCs to initiate and orchestrate innate and 99 specific immune responses.

100

101

2. Materials and Methods

102

103

\subsection{Animals}

Specific pathogen free (SPF) channel catfish fingerlings (five-six month old) and fry (one

105 month old) were acquired from the fish hatchery at the College of Veterinary Medicine,

106 Mississippi State University and maintained into flow-through tanks at $25-28{ }^{\circ} \mathrm{C}$. Fish were fed

107 with a floating catfish feed to satiety and acclimated for one week. In this present study, all fish

108 experiments were carried out by a protocol approved by the Institutional Animal Care and Use

109 Committee (IACUC) at Mississippi State University. The fish were euthanized in water

110 containing $100 \mathrm{mg} / \mathrm{L}$ tricaine methanesulfonate (MS-222, Western, Chemical, Inc.). Samples

111 were obtained as described below.

112

113

2.2. Cell Preparation

114

Peripheral blood, anterior kidney, and spleen cell separation was performed as described

115 previously [17]. Briefly, peripheral blood was collected from the caudal vein followed by

116 centrifugation at $500 \mathrm{~g}$ and resuspended in Phosphate-buffered saline (PBS). Anterior kidney and

117 spleen were dissected from the fish and placed in a sterile culture dish containing PBS. To obtain

118 a single-cell suspension, tissue were minced with sterile forceps, repeatedly aspirated using a 1

119 ml syringe and passed through cell dissociation sieves (Sigma, St. Louis, MO). The resulting cell

120 suspensions were washed and resuspended in PBS. Cell suspensions and peripheral blood were 
121 layered over Histopaque 1077 (Sigma) and centrifuged at 500g for $30 \mathrm{~min}$. Cells then were

122 collected from the interface, washed three times in PBS, counted and assessed for viability by

123 trypan blue exclusion.

124

125

2.3. Cell Staining and Flow Cytometry

126

127 (PE Mouse Anti-human L/CD207, clone no: 2G3, BD Biosciences) and isotype-matched

128 controls (Ig Lambda, clone no: 1-155-2, eBioscience) were used to identify the intracellular

129 staining of L/CD207 in the immune related organs of catfish. Cells from peripheral blood, spleen

130 and anterior kidney were fixed and permeabilized with BD Perm/Wash buffer (BD Bioscineces)

131 on ice for $20 \mathrm{~min}$, and washed twice with BD Perm/WashTM buffer (BD Biosciences).

132 Following fixation and permeabilization, cells were re-suspended with Streptavidin/Biotin

133 (Vector Laboratories) and incubated for $15 \mathrm{~min}$ in the dark and washed with BD Perm/WashTM

134 buffer. After that, cells were incubated with R-Phycoerythrin-conjugated mAbs to human

$135 \mathrm{~L} / \mathrm{CD} 207$ on ice for $30 \mathrm{~min}$ in the dark, and washed with BD Perm/WashTM buffer. Finally,

136 cells were incubated with Streptavidin R-Phycoerythrin Conjugate (Life Technologies) on ice for

$13730 \mathrm{~min}$ in the dark and washed with BD Perm/WashTM buffer.

138 Catfish mononuclear cells were gated based on their relative size and granularity using

139 forward and side scatters (FSC and SSC, respectively) with a FACSCalibur Flow Cytometer

140 (Becton Dickinson). Immunofluorescent staining was analyzed using FlowJo 7.6.4 Software

141 (Tree Star Inc.). The intensity of L/CD207 staining in catfish mononuclear cells was analyzed by

142 using single histogram statistics. 


\subsection{Tissue Preparation for Immunohistochemistry}

Spleen, anterior kidney, gill, and skin were isolated from the euthanized channel catfish and immediately fixed in $10 \%$ neutral buffered formalin for $24 \mathrm{~h}$. Tissues were embedded in paraffin wax after the dehydration with a graded series of ethanol, and 5 or 6 sections were cut for per slide as described [14].

\subsection{Immunohistochemistry (IHC)}

Immunohistochemical staining of fingerling catfish spleen, anterior kidney, skin and gill paraffin slides for the presence of $\mathrm{L} / \mathrm{CD} 207^{+}$cells was performed with two different antibodies: purified human CD207-specific pAbs (R\&D Systems, Inc.) and R-Phycoerythrin-conjugated mAbs specific to human L/207. Immunohistochemical staining with purified pAbs was conducted as described elsewhere with minor modifications [15]. In this study, antigen retrieval was examined by incubation of sections in target retrieval solution (DAKO) for $40 \mathrm{~min}$ at $100^{\circ} \mathrm{C}$. Following this incubation, sections were incubated in protein block (DAKO) for $1 \mathrm{~h}$. After that, primary antibody was diluted at a concentration of $0.2 \mathrm{mg} / \mathrm{ml}$ (1:500 from stock solution), and sections were incubated in primary antibody for overnight at room temperature in a humid chamber. We used rainbow trout spleen as a positive control for L/CD207-specific staining. Also, negative control that was normal goat IgG (Vector Laboratories) was applied at the same concentrations as the primary antibody. After overnight incubation, streptavidin/biotin blocks were applied for 15 min. Finally, sections were incubated in Streptavidin-HRP (Vector Laboratories) for one hour. Then, slides were analyzed and photographed with OLYMPUS BX43 microscopy and cell Sens standard software program. 
Immunohistochemistry with conjugated mAbs was performed as described for purified

167 pAbs. However, there were some differences such as: $0.3 \%$ hydrogen peroxide in $100 \%$

168 methanol were not used in alcohol serial dilutions, and sections were incubated with primary

169 conjugated antibody for four hours and washed in PBS for $10 \mathrm{~min}$. Then, slides were analyzed by

170 Immunofluorescent Microscopy (CYTATION 5 imaging reader, BioTek).

171

172 2.6. Transmission Electron Microscopy (TEM)

173 Sample preparation for TEM was carried out as described [14]. Spleen and posterior

174 kidney samples were fixed in McDowell's fixative. After primary fixation, samples were rinsed

175 and postfixed in $2 \%$ buffered Osmium tetroxide, dehydrated through a graded ethanol series, and

176 embedded in Suprr's resin. Ultra-thin sections were cut on a Reichert-Jung Ultracut E

177 ultramicrotome, and then sections were stained with uranyl acetate and lead citrate. Sections

178 were viewed using a JEOL JEM1230 Transmission Electron microscope at 80kv equipped with 179 an AMT XR50 camera.

180

181

182

\subsection{Statistical analysis}

Analysis of variance (ANOVA), followed by Fisher's LSD multiple comparison post hoc

183 test was used to evaluate differences in mean fluorescence intensity (MFIs). The level of

184 significance for all tests was set at $\mathrm{P}<0.05$.

185

186

187

188 


\section{Results}

190

3.1. Assessment of Langerin/CD207 ${ }^{+}$cells in immunocompetent tissues of channel catfish by

192 flow cytometry

193 To identify the presence of $\mathrm{L} / \mathrm{CD} 207^{+}$LC-like cells in the immunocompetent organs of channel

194 catfish mononuclear cells derived from spleen, anterior kidney, and peripheral blood were 195 analyzed for the intensity of staining with human L/CD207+- specific mAbs. The levels of

196 L/CD207 expression in mononuclear cells obtained from 7 fingerling catfish were low to 197 negative in anterior kidney and spleen, and virtually negative in the peripheral blood (data not

198 shown). There were no statistically significant differences in the intensity of L/CD207 staining of 199 the spleen and anterior kidney mononuclear cells compared to the isotype-matched controls $200 \quad(\mathrm{P}>0.05)$.

201

202 3.2. Identification of Langerin $/ C D 207^{+}$cells in immunocompetent tissues of channel catfish by $203 I H C$

204 To confirm our data on a possible presence of LC-like L/CD207 ${ }^{+}$cells in the 205 immunocompetent organs of catfish by flow cytometry, we have adapted the IHC protocol that 206 was previously described for identification of the $\mathrm{L} / \mathrm{CD} 207^{+}$cells in rainbow trout and salmonid 207 [15]. Visually strong L/CD207 cytoplasmic expression was observed in the spleen and anterior 208 kidney tissues compared to virtually undetected reaction in the negative controls (Fig. 1 A, B, C $209\{$ Panels I, II $\}$ ). Interestingly, the L/CD207 ${ }^{+}$cells in the anterior kidney were more abundant 210 compared to their counterparts in the spleen and relatively rare in the gill (Fig. 1 D \{Panels I, 211 II $\}$ ). Notably, the numbers of L/CD207 ${ }^{+}$varied significantly in pathogen free fingerlings, from 
212 just a few cells to the cell numbers shown in Figure I C. However, the skin of channel catfish

213 was constantly negative for L/CD207 (data not shown).

Spleen, anterior kidney, gill and skin tissues were examined by IHC using R-PE

215 conjugated mAbs specific to human L/CD207 and the isotype-matched control mAbs to

216 document the presence of $\mathrm{L} / \mathrm{CD} 207^{+}$cells in the tissues of catfish. Langerin-positive cells have

217 been identified mostly in catfish anterior kidney confirming our findings obtained by flow

218 cytometry and immunohistochemistry using purified polyclonal L/CD207-specific Abs (Fig. 2).

219 Importantly, the $\mathrm{L} / \mathrm{CD} 207^{+}$cells in the spleen were concentrated around blood vessels or

220 sinusoids (Fig. 2). Contrary, gill and skin tissues showed the background intensity of L/CD207

221 staining comparable with negative controls (data not shown).

222

223 vaccinated catfish fingerling and fry, and the ultrastructure of these DL cells was analyzed by

\subsection{Identification of Dendritic-like cells in lymphoid tissue of channel catfish by TEM}

Dendritic-like (DL) cells were observed in the immunologically important organs of nonTEM (Fig. 3A, B). Dendritic-like cells contained an unsegmented nucleus and did not have long cytoplasmic processes. These cells possessed Birbeck-Like (BL) granules with tennis-racket or rod-shaped morphology. Birbeck-Like (BL) granules that were not a continuation of the cell

229 membrane were found surrounding the centriole in the cytoplasm of the DL cells.

\section{Discussion}

The aim of the current research was to assess morphologically the most powerful professional APCs, DCs in immune related organs of channel catfish. We identified L/CD207 ${ }^{+}$ 
235 cells in the spleen, anterior kidney, and gill of channel catfish. Importantly, the L/CD207 ${ }^{+}$DCs

236 visually prevailed in the catfish anterior kidney compared to their counterparts in the spleen.

237 Also, the $\mathrm{L} / \mathrm{CD} 207^{+}$cells were virtually absent in the skin and rare in the gill of catfish.

$238 \mathrm{~L} / \mathrm{CD} 207^{+}$were firstly described in the spleen and anterior kidney of Atlantic salmon and

239 rainbow trout with remarkably similarities to human LCs [15]. Unlike in channel catfish,

$240 \mathrm{~L} / \mathrm{CD} 207^{+}$were observed in a fewer numbers in the anterior kidney compared to the spleen of

241 Atlantic salmon and rainbow trout and like in catfish, were not detected in the skin [15]. These

242 results show that anterior kidney and spleen in channel catfish serve as important organs for

243 antigen presentation as was described previously for rainbow trout, salmon and zebrafish [2, 5,

244 15]. Interestingly, $\mathrm{L} / \mathrm{CD} 207^{+}$cells in teleost fish were found predominantly in the spleen and

245 anterior kidney as LCs in mammals populate mainly the epidermis of skin and in the process of

246 maturation migrate to the secondary lymphoid organs [18]. In particular, we were able to

247 document the presence of $\mathrm{L} / \mathrm{CD} 207^{+} \mathrm{DCs}$ in the fingerling spleen located in close proximity to

248 sinusoids or blood vessels suggesting that catfish LCs can sample and capture antigens

249 trafficking through the blood with subsequent maturation and presentation of processed antigens

250 to immune competent T cells in the spleen.. Interestingly, a single L/CD $207^{+}$identified in

251 catfish gill was located near the blood vessel suggesting possible sentinel role of fish DCs in

252 sampling antigens in the periphery and trafficking them to the lymphoid organs. As fish lack

253 lymph nodes, the migration pattern and maturation of DCs might be different from humans and

254 other mammals. Dendritic-like populations identified in the immunocompetent organs of catfish

255 may share morphological and functional properties with previously reported DCs in teleost fish

256 and mammals. 
We described BL granules in DC-like cells of catfish spleen and posterior kidney by transmission electron microscopy. The granules had a rod portion and were vacuolated at one end that gave the organelles a tennis-racket morphology that was described previously in Atlantic salmon and zebrafish strikingly resembling human LCs [2, 13, 14]. Birbeck-like granules with rod-shaped and tennis-racked morphology were observed for the first-time in fish

262 within the diseased gills of Chinook salmon (Oncorhynchus tshawytscha) and lymphoid organs 263 of salmonids $[13,14]$. Lately, BL granules were also detected in the DCs isolated from the skin 264 of zebrafish [2]. Importantly, the unique location of these granules surrounding the centriole in 265 the cytoplasm of the DL cells in the spleen of catfish was very similar to the location in salmonids $[13,14]$. Furthermore, BGs in LCs were commonly found near centrioles and

267 microtubules in patients with 'atypical hyperplasia' in humans [19]. It was suggested that BGs in 268 humans have a function of a loading compartment for antigens before LCs undergo maturation 269 and migration to the secondary lymphoid organs [20]. Therefore, centrioles and microtubules are 270 necessary during antigen processing in immature DCs [21]. Despite of the lacking information 271 on the ultrastructural details of BL granules and accumulation of Langerin in their close 272 proximity, our study identified BL granules in catfish that are virtually identical to BGs 273 described in Dc-like cells of salmonids and zebrafish, and also LCs in humans.

274 Langerin protein and BL granules, the hallmarks of mammalian LCs were detected in the 275 immunocompetent organs of catfish, suggesting that teleost fish, the earliest vertebrates that 276 develop adaptive immunity possess LC-like cells. The LC-like cell populations in catfish may 277 represent an earlier lineage to Langerhans cells that are found within the epidermis in humans 278 and other mammals. In conclusion, we have identified LC-like cells in catfish based on the 279 expression of Langerin by immunohistochemistry and flow cytometry and their unique 
morphology by TEM. However, some questions still remain unanswered. Is the Langerin identified by using human-specific antibodies the only type of protein present in fish or do other

282 isoforms that are fish-specific and could not be detected by human-specific antibodies exist? We

283 also cannot rule out that several LC-like populations in catfish are present.

284 More detailed knowledge about the functional properties of the catfish Langerin ${ }^{+}$cells, their role 285 as professional APCs in innate and adaptive immunity will not only help gain insight into the 286 evolution of the vertebrate adaptive immune system but will provide valuable information for 287 development and optimization of immunotherapies and vaccination protocols for aquaculture 288 use.

\section{Conflict of Interest Statement}

291 The authors have declared no competing interests.

Acknowledgements

The funding for this research was provided by an internal research grant from the College of Veterinary Medicine Mississippi State University awarded to LMP, AK, WB.

297 that served as positive controls in the IHC experiments. The flow cytometry data were acquired 298 by the Mississippi State University Basic Sciences Flow Cytometry Core Facility. All tissue 299 slides have been prepared in the College of Veterinary Medicine Histology Laboratory. The 300 TEM data were acquired Institute for Imaging \& Analytical Technologies. We would like to 301 acknowledge the assistance of Dr. Pharr, Courtney Hunter, Dr. Park, Amanda Lawrence and the 302 staff members of Dr. Petrie-Hanson's Laboratory. 


\section{References}

[1] A. Mildner, S. Jung, Development and function of dendritic cell subsets, Immunity 40(5) (2014) 642-56.

[2] G. Lugo-Villarino, K.M. Balla, D.L. Stachura, K. Banuelos, M.B. Werneck, D. Traver, Identification of dendritic antigen-presenting cells in the zebrafish, Proc Natl Acad Sci U S A 107(36) (2010) 15850-5.

[3] V. Wittamer, J.Y. Bertrand, P.W. Gutschow, D. Traver, Characterization of the mononuclear phagocyte system in zebrafish, Blood 117(26) (2011) 7126-35.

[4] N. Aghaallaei, B. Bajoghli, H. Schwarz, M. Schorpp, T. Boehm, Characterization of mononuclear phagocytic cells in medaka fish transgenic for a cxcr3a:gfp reporter, Proc Natl Acad Sci U S A 107(42) (2010) 18079-84.

[5] E. Bassity, T.G. Clark, Functional identification of dendritic cells in the teleost model, rainbow trout (Oncorhynchus mykiss), PLoS One 7(3) (2012) e33196.

[6] A.F. Lin, L.X. Xiang, Q.L. Wang, W.R. Dong, Y.F. Gong, J.Z. Shao, The DC-SIGN of zebrafish: insights into the existence of a CD209 homologue in a lower vertebrate and its involvement in adaptive immunity, J Immunol 183(11) (2009) 7398-410.

[7] T. Shao, L.Y. Zhu, L. Nie, W. Shi, W.R. Dong, L.X. Xiang, J.Z. Shao, Characterization of surface phenotypic molecules of teleost dendritic cells, Dev Comp Immunol 49(1) (2015) 3843.

[8] B.Z. Igyarto, D.H. Kaplan, Antigen presentation by Langerhans cells, Curr Opin Immunol 25(1) (2013) 115-9. 
324 [9] K. Sugita, K. Kabashima, K. Atarashi, T. Shimauchi, M. Kobayashi, Y. Tokura, Innate

325 immunity mediated by epidermal keratinocytes promotes acquired immunity involving

326 Langerhans cells and T cells in the skin, Clin Exp Immunol 147(1) (2007) 176-83.

327 [10] J. Valladeau, C. Dezutter-Dambuyant, S. Saeland, Langerin/CD207 sheds light on formation

328 of birbeck granules and their possible function in Langerhans cells, Immunol Res 28(2)

$329 \quad$ (2003) 93-107.

330 [11] J. Valladeau, O. Ravel, C. Dezutter-Dambuyant, K. Moore, M. Kleijmeer, Y. Liu, V.

331 Duvert-Frances, C. Vincent, D. Schmitt, J. Davoust, C. Caux, S. Lebecque, S. Saeland,

332 Langerin, a novel C-type lectin specific to Langerhans cells, is an endocytic receptor that

333 induces the formation of Birbeck granules, Immunity 12(1) (2000) 71-81.

334 [12] C.M. Fehres, S.C. Bruijns, B.N. Sotthewes, H. Kalay, L. Schaffer, S.R. Head, T.D. de

335 Gruijl, J.J. Garcia-Vallejo, Y. van Kooyk, Phenotypic and Functional Properties of Human

336 Steady State CD14+ and CD1a+ Antigen Presenting Cells and Epidermal Langerhans Cells,

337 PLoS One 10(11) (2015) e0143519.

338 [13] J. Lovy, G.M. Wright, D.J. Speare, Morphological presentation of a dendritic-like cell

339 within the gills of chinook salmon infected with Loma salmonae, Dev Comp Immunol 30(3)

$340 \quad$ (2006) 259-63.

341 [14] J. Lovy, G.M. Wright, D.J. Speare, Comparative cellular morphology suggesting the

342 existence of resident dendritic cells within immune organs of salmonids, Anat Rec

343 (Hoboken) 291(4) (2008) 456-62.

344 [15] J. Lovy, G.P. Savidant, D.J. Speare, G.M. Wright, Langerin/CD207 positive dendritic-like

345 cells in the haemopoietic tissues of salmonids, Fish Shellfish Immunol 27(2) (2009) 365-8. 
[16] USDA, Catfish database, National Agricultural Statistics Service, A.S.B., United States Department of Agriculture in: e. http://www.agecon.msstate.edu/whatwedo/budgets/docs/catfish2014.pdf (Ed.) 2014.

[17] X. Zhao, R.C. Findly, H.W. Dickerson, Cutaneous antibody-secreting cells and B cells in a teleost fish, Dev Comp Immunol 32(5) (2008) 500-8.

[18] F. Geissmann, M.C. Dieu-Nosjean, C. Dezutter, J. Valladeau, S. Kayal, M. Leborgne, N. Brousse, S. Saeland, J. Davoust, Accumulation of immature Langerhans cells in human lymph nodes draining chronically inflamed skin, J Exp Med 196(4) (2002) 417-30.

[19] M. Shamoto, M. Hoshino, T. Suchi, Cells containing Langerhans cell granules in human lymph nodes of "atypical hyperplasia" with fatal outcome and leukemic reticuloendotheliosis, Acta Pathol Jpn 26(3) (1976) 311-23.

[20] R. Mc Dermott, U. Ziylan, D. Spehner, H. Bausinger, D. Lipsker, M. Mommaas, J.P. Cazenave, G. Raposo, B. Goud, H. de la Salle, J. Salamero, D. Hanau, Birbeck granules are subdomains of endosomal recycling compartment in human epidermal Langerhans cells, which form where Langerin accumulates, Mol Biol Cell 13(1) (2002) 317-35.

[21] K.K. Peachman, M. Rao, D.R. Palmer, M. Zidanic, W. Sun, C.R. Alving, S.W. Rothwell, Functional microtubules are required for antigen processing by macrophages and dendritic cells, Immunol Lett 95(1) (2004) 13-24. 


\section{Figure legends}

367 Fig. 1. Assessment of $\mathrm{L} / \mathrm{CD} 207^{+}$cells in the spleen, anterior kidney, and gill of catfish fingerling 368 by immunohistochemical staining with purified pAbs specific to human Langerin.

369 Photomicrographs (600X). Panels (I) show L/CD207-specific staining with pAbs in different 370 concentrations: A) Catfish spleen, Abs dilution 1:50; B) Catfish spleen, Abs dilution 1:500; C)

371 Catfish anterior kidney, Abs dilution 1:500; D) Catfish gill, Abs dilution1:500; E) Rainbow trout 372 spleen (positive control), Abs dilution 1:50. Panels (II) show negative controls (normal goat IgG) 373 catfish spleen, anterior kidney, gill, and rainbow trout spleen, respectively. Exerts on the left 374 show magnified images of the selected areas.

375

376 Fig. 2. Assessment of $\mathrm{L} / \mathrm{CD} 207^{+}$cells in the anterior kidney of catfish fingerling by 377 immunohistochemical staining with R-PE-conjugated mAbs specific to human Langerin.

378 Photomicrographs (600X). Panel (I) shows Langerin-specific staining. Panel (II) shows tissue 379 staining with matching R-PE-conjugated isotype control mAbs (negative control). Panel (III) 380 shows magnified images of the selected areas. Arrows identify Langerin-specific binding with 381 red fluorescence.

383 Fig. 3. Dendritic-Like (DL) cell in the immune-related organs of Ictalurus punctatus by electron 384 microscopy. DL cell with unsegmented nucleus $(\mathrm{N})$ and Birbeck-like (BL) granules (solid arrow) 385 in the cytoplasm surrounding the centriole (dotted arrow). (A) Spleen, fingerling; (B) Anterior 386 kidney, fry; (C) Posterior kidney, fry; (D) Gill, fry. 
Figure 1

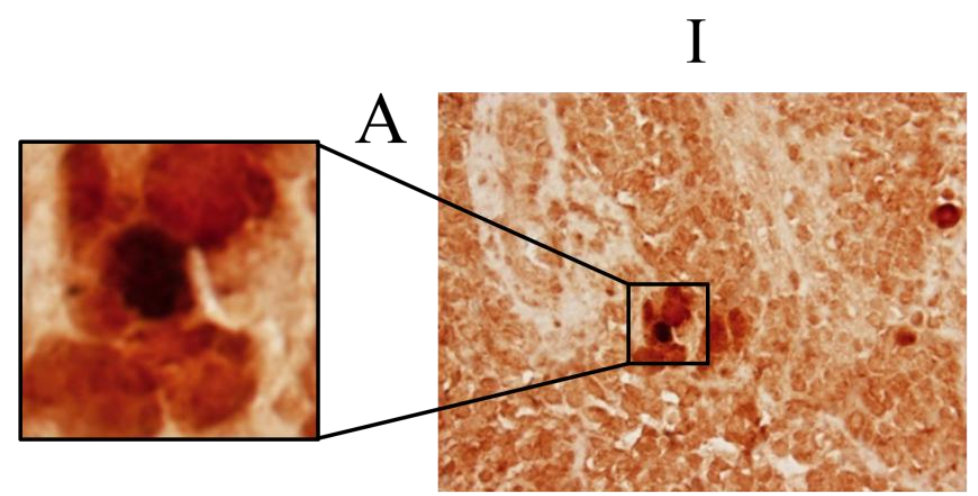

II
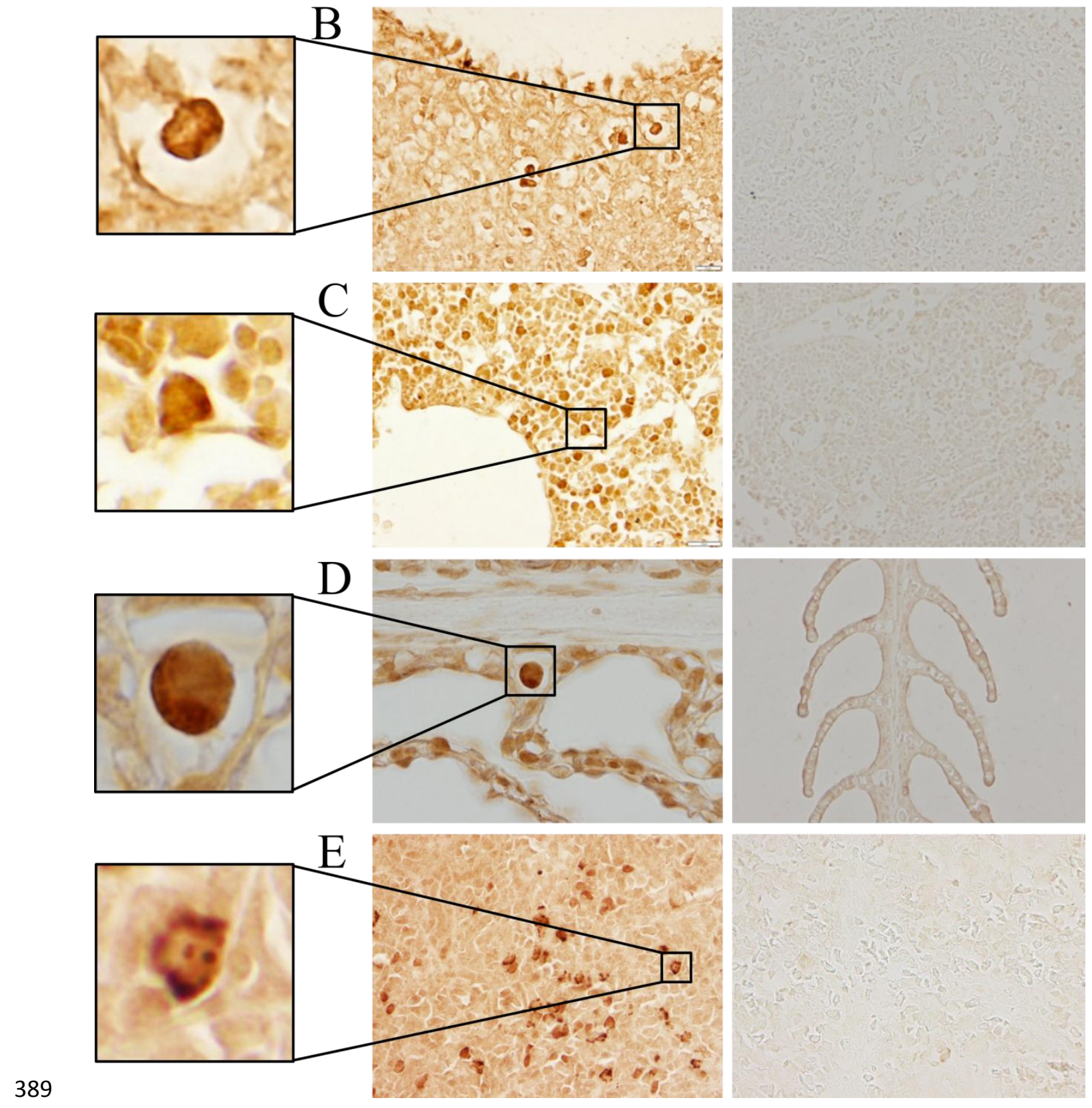
390 Figure 2.

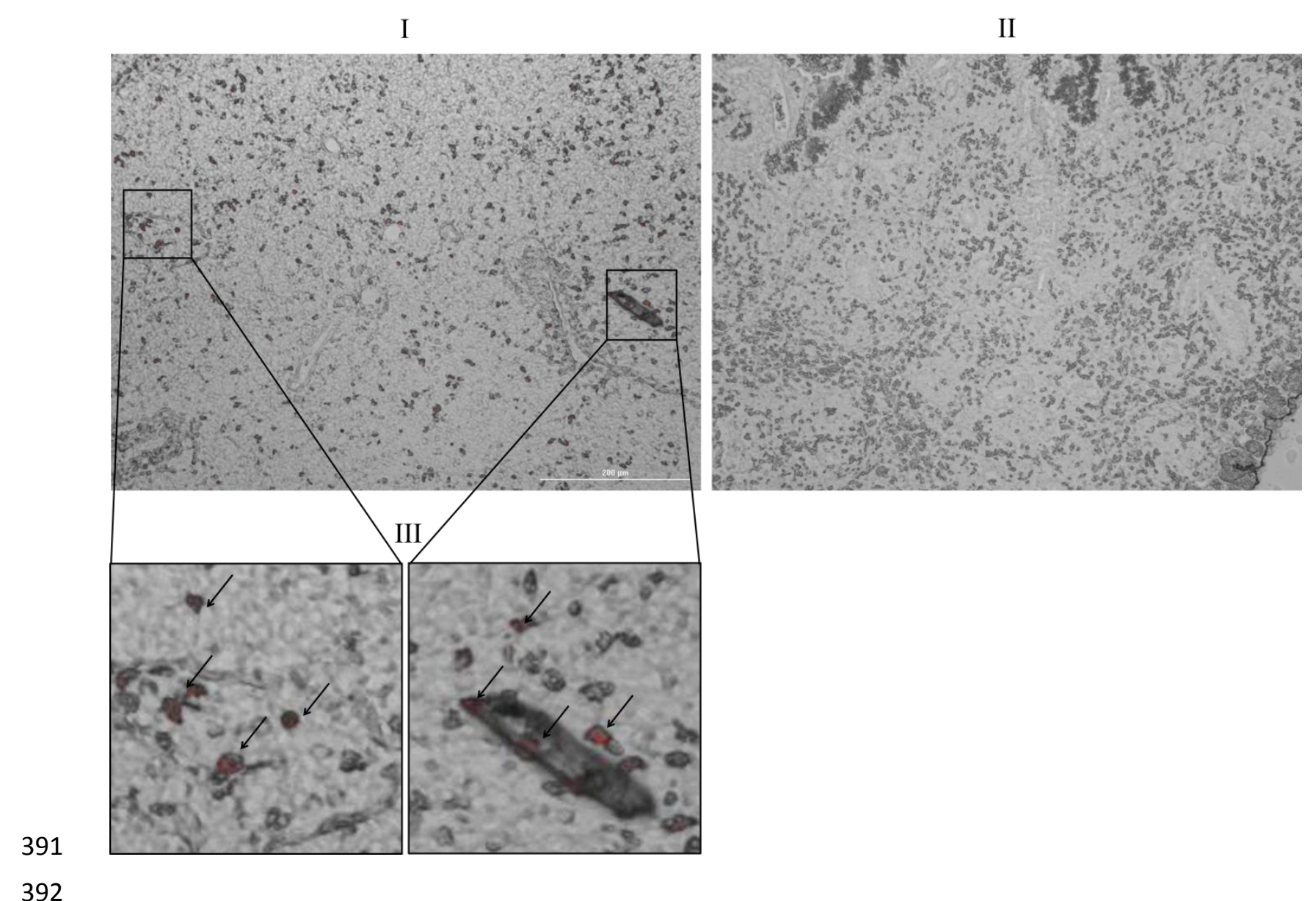

392 
$393 \quad$ Figure 3

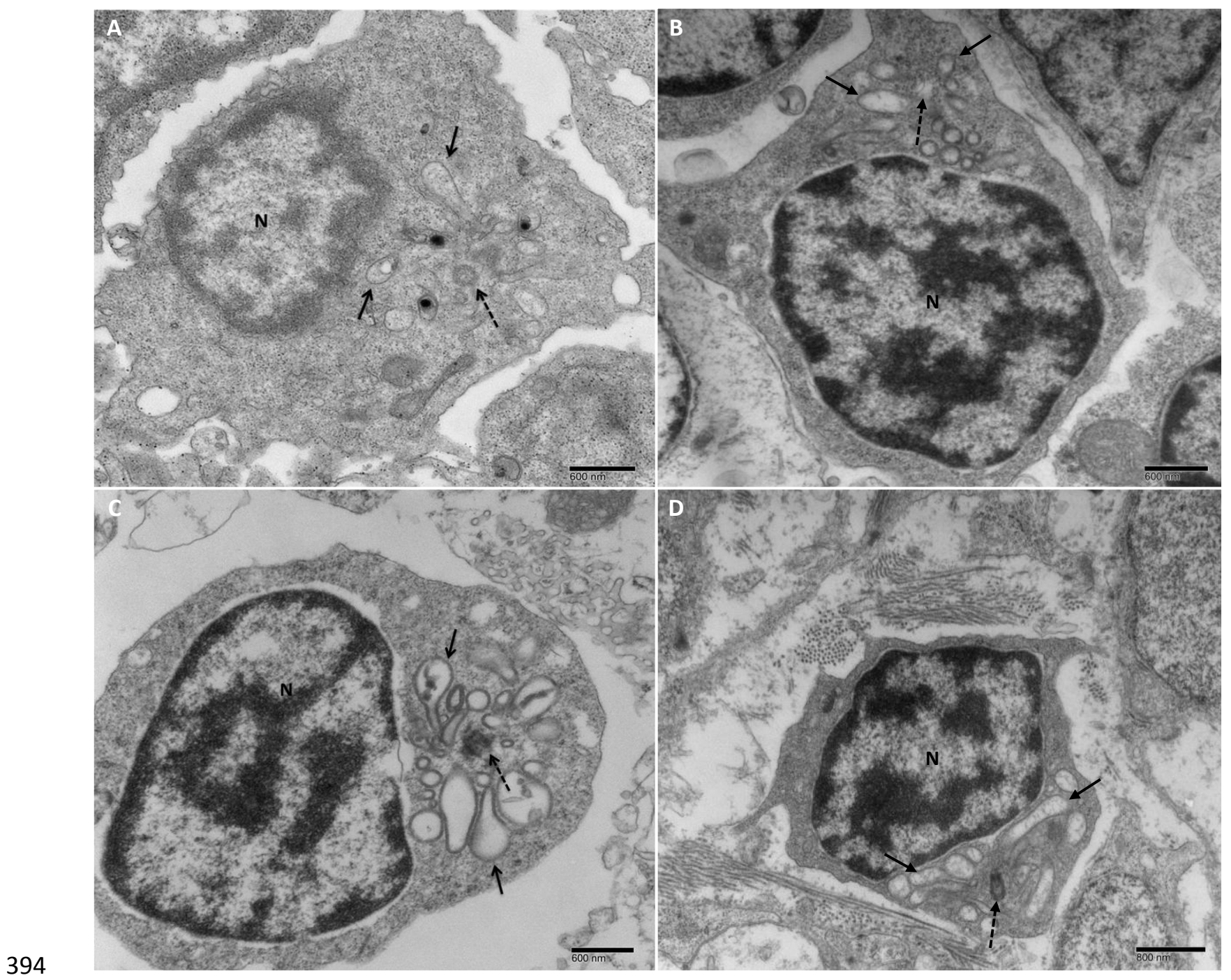

395 